\title{
Respuesta fisiológica y molecular de Anadara tuberculosa (Arcoida: Arcidae) al estrés de salinidad
}

\author{
Oscar Mendoza ${ }^{1}$, Krizia Pretell ${ }^{1}$, Benoit Diringer ${ }^{2,4}$, Ricardo Avellan $^{3}$, Karina Zapata ${ }^{2}$, \\ Angelita Marchan ${ }^{1}$, Virna Cedeño ${ }^{1,3}$, Tessy Peralta ${ }^{1}$, Alberto Ordinola ${ }^{1}$ \& Eric Mialhe ${ }^{2,3}$ \\ 1. Facultad de Ingeniería Pesquera y Ciencias del Mar, Universidad Nacional de Tumbes, Perú; \\ omendozan@untumbes.edu.pe, krizmar_5@hotmail.com, tauro_27_193@hotmail.com, tesymar765@gmail.com, \\ alordin@gmail.com, karinazapatavidaurre@gmail.com \\ 2. Inca Biotec SAC, Perú; diringerb@yahoo.fr, ericmialhe@yahoo.fr \\ 3. Concepto Azul AS, Ecuador; ravellan21@yahoo.com,virna.cedenoescobar@gmail.com \\ 4. EPHE, Pathologie Comparée des Invertébrés, Montpellier (France).
}

Recibido 27-VIII-2016. Corregido 27-IV-2017. Aceptado 31-V-2017.

\begin{abstract}
Physiological and molecular response of Anadara tuberculosa (Arcoida: Arcidae) to salinity stress. The pustulose ark A. tuberculosa is an emblematic species of mangrove ecosystem that is currently in a vulnerable condition. The development of its aquaculture, to begin with genetic breeding programs, requires the identification of molecular biomarkers, particularly those associated with salinity stress. With this purpose, specimens of A. tuberculosa were collected from the adjacent mangroves of Puerto Pizarro bay (Tumbes, Perú), from January 2015 to February 2016. Different assays (groups of ten animals in triplicate) were undertaken in separated periods of 16 days: hypo-osmotic stress (extreme: 5, $10 \mathrm{ppt}$ ); (Moderate: 15, $25 \mathrm{ppt}$ ) and no stress (control group: $33 \mathrm{ppt}$ ). The presence of salinity stress biomarkers was assessed at the genetic level throughout PCR detection of 19 genes reported to be key actors in osmoregulation, and at the proteomic level with the sequencing of peptides (tandem mass spectrometry MALDI TOF/TOF), expressed in ark tissues exposed to different salinities. None of the tested genetic markers could be amplified by PCR, suggesting that $A$. tuberculosa has significant genetic differences compared to other mollusks. Proteomic analysis by mass spectrometry on $A$. tuberculosa gill tissue, allowed to identify 26 peptides expressed in presential and differential forms at different salinities, highlighting possible markers such as HSP70 and trans-membrane chloride channel transportation protein, to be related with salinity adaptation. These amino acid sequences will allow the design of target specific primers for A. tuberculosa, to implement future research in ecophysiology of this important fishery resource. Rev. Biol. Trop. 65 (3): 1142-1151. Epub 2017 September 01.
\end{abstract}

Key words: Anadara tuberculosa, osmoregulation, salinity, adaptation, mangrove.

Anadara tuberculosa (Sowerby, 1833), popularmente conocida en la región Tumbes como concha negra, es un molusco bivalvo perteneciente a la familia Arcidae, que se distribuye a lo largo de la costa del Océano Pacífico, desde Baja California, México hasta Tumbes, Perú (Espinosa, Delgado, Orobio, Mejía-Ladino, \& Gil-Agudelo, 2010; Cruz, Fonseca, \& Chavarría-Solera, 2012); habita la zona intermareal, asociada a los sustratos fangosos, arcillosos o limo-arcillosos del ecosistema de manglar (Mendoza \& Peralta, 2004). Se explota artesanalmente por extractores conocidos como "concheros" quienes sustentan parte de su economía con la extracción y venta de este molusco; sin embargo, la mayoría de los bancos naturales actualmente se encuentran sobreexplotados y algunas poblaciones, en la mayoría de los países, cercanas al colapso (Lucero, Cantera, \& Neira, 2012). En Perú, las poblaciones de $A$. tuberculosa se han reducido en un $73.2 \%$ durante el periodo de 1996 al 2007 (Ordinola, Montero, Alemán, \& Llanos, 2007). Por este motivo, se están realizando 
actividades alternativas como la producción de semillas en laboratorio, cuyas experiencias favorables han sido posibles en México, El Salvador, Perú y Ecuador. En Perú, el engorde en el manglar de semillas producidas en laboratorio ha sido un éxito, aunque con problemas de elevadas mortalidades, asociadas al descenso de la salinidad, en los meses de lluvia (Diringer, Vásquez, Moreno, Pretell, \& Sahuquet, 2012; Pretell, 2015-comunicación personal).

A pesar de las diferencias de sensibilidad a variaciones de salinidades, todos los bivalvos son osmoconformes, es decir poseen poca o nula capacidad para la regulación osmótica de su hemolinfa, motivo por lo que, las células soportan la carga de la regulación del volumen ajustando las concentraciones intracelulares de amino ácidos libres (FAAs) y otras moléculas orgánicas pequeñas; sin embargo, los FAAs son principalmente obtenidos del catabolismo de proteínas (autofagia) cuya excesiva pérdida provoca la muerte del animal (Gosling, 2015). A. tuberculosa adultas, muestran gran adaptación a salinidades de 20 a 40 ppt, sin mortalidades asociadas (Nieves et al., 2009); pero experiencias realizadas sobre semillas de $5 \mathrm{~mm}$, mostraron que a salinidades inferiores a 25 ppt provocan importantes mortalidades después de unos días (Diringer et al., 2012).

Los principales mecanismos de adaptación a diferentes salinidades de la ostra y del mejillón han sido caracterizados a nivel molecular (Cross, Merlo, Rodríguez, Portela-Bens, \& Rebordinos, 2014; Eierman \& Hare, 2014); en particular, Meng et al. (2013), resaltaron la primordial importancia de las vías de metabolismo de los FAAs para la adaptación eurihalina de la ostra Crassostrea gigas. A pesar que la mayoría de los trabajos han sido realizados en diferentes especies de bivalvos, es muy probable que los mecanismos de osmorregulación se conserven a nivel inter-específico. Recientemente, tecnologías de proteómica se han presentado como una alternativa para estudiar la respuesta de bivalvos frente a estrés abióticos (Artigaud et al., 2015; Rocher et al., 2015).

Por lo anterior, es que el objetivo de este trabajo fue determinar la respuesta fisiológica y molecular de $A$. tuberculosa sometida a un estrés de baja salinidad. Adultos de A. tuberculosa fueron sometidos a salinidades de 5 a 33 ppt mediante desafíos experimentales de diferentes rangos de salinidad. Asimismo, en paralelo se caracterizó los genes asociados a la osmorregulación con técnicas de genómica y de proteómica comparativas, permitiendo caracterizar la respuesta de concha negra al estrés de salinidad, tanto en lo ecofisiológico como en lo molecular, para entender los mecanismos de adaptación, que permitirán a futuro seleccionar marcadores para programas de mejoramiento genético de este importante recurso en la Región Tumbes.

\section{MATERIALES Y MÉTODOS}

Un total de 150 ejemplares de $A$. tuberculosa fueron recolectados entre agosto $2015 \mathrm{y}$ enero 2016 en los bancos naturales del manglar colindante a la Bahía de Puerto Pizarro (330'12.96” S - 80²3'55.87' W), Tumbes, Perú. Los esteros que conforman este ecosistema poseen una salinidad de 33 ppt en marea alta, llegando a alcanzar una altura promedio de $1.20 \mathrm{~m}$, con una fluctuación entre la alta y baja marea de 6 horas. Se seleccionaron los ejemplares que presentaban un tamaño $\geq 45 \mathrm{~mm}$ de longitud de valva para luego ser transportados al Centro Colectivo Educativo Experimental de Biología y Biotecnología Acuática y Acuícola de Puerto Pizarro (CEBAP).

Aclimatación y desafíos experimentales: Se realizó un primer ensayo en agosto 2015. La parte ventral de la concha de los ejemplares seleccionados fue limada con la finalidad de que las branquias queden expuestas (Zhao, Yu, Kong, \& Li, 2012). Posteriormente, todos ellos se aclimataron por 24 horas a 33 ppt de salinidad en agua de mar filtrada a $1 \mu \mathrm{m}$ y tratada (AMFT) con 10 ppm de $\mathrm{Ca}(\mathrm{ClO})_{2}$ y neutralizada con $0.9 \mathrm{ppm}$ de $\mathrm{Na}_{2} \mathrm{~S}_{2} \mathrm{O}_{3}$. Transcurrido este periodo, se formaron grupos de diez animales por triplicado y se colocaron en recipientes con 20 litros de agua de mar previamente mezclada con diferentes proporciones de agua dulce 
para obtener los diferentes grados de salinidad. Los individuos fueron directamente sometidos a condiciones de estrés hipo-osmótico (extremo: 5 y 10 ppt); (moderado: 15 y 25 ppt) y sin estrés (grupo control: $33 \mathrm{ppt)}$ ). Las muestras fueron evaluadas por un periodo de 16 días con $100 \%$ de recambio de agua cada 48 h, o en caso de presentar mortalidad. Los animales fueron alimentados durante el tiempo del bioensayo con un mix de microalgas (Isocrisis sp. y Chaetoceros spp.) a una concentración de $50000 \mathrm{cel} / \mathrm{mL}$ de cada cepa, una vez al día. Estas muestras sirvieron para las evaluaciones genéticas.

En enero 2016, se realizó una segunda prueba utilizando el mismo procedimiento antes mencionado para la evaluación proteómica, con la diferencia de que los animales fueron expuestos solamente por periodos de 4,8 y 12 horas, sin alimentación.

Extracción de ADN: Se usó $80 \mathrm{mg}$ de tejido branquial macerado de $A$. tuberculosa, mediante el método de extracción CTAB $2 \%$ (Bromuro de hexadeciltrimetilamonio), complementado con proteinasa K (Gardes \& Bruns, 1993). El ADN genómico se obtuvo con fenol, cloroformo y alcohol isoamil en proporción $(25: 24: 1 \mathrm{v} / \mathrm{v})$. Se precipitó con etanol frío al $95 \%$, y se realizó un lavado con etanol al 75 $\%$. El ADN obtenido se resuspendió en $50 \mu \mathrm{L}$ de buffer TE Tris/EDTA (Tris $10 \mathrm{mM}$, EDTA 1 $\mathrm{mM})$ y fue conservado a $-20{ }^{\circ} \mathrm{C}$.

Amplificación por PCR de genes de referencia y genes de interés: La calidad del ADN genómico se evaluó por amplificación del gen constitutivo mitocondrial COI con los iniciadores LCO (GGTCAACAAATCATAAAGATATTGG') y HCO (TAAACTTCAGGG TGACCAAA AAATCA). Los genes de interés evaluados para este estudio fueron: Aminomethyltransferase, Glycine dehydrogenase, Glycine hydroxymethyltransferase, $\Delta-1$ pyrroline-5-carboxylate synthase, Ornithine aminotransferase, Arginase, Alanine transaminase, Alanine-glyoxylate transaminase, Glutamate decarboxylase, Glutamate decarboxylase
1-2, Spermidine synthase, Aldehyde dehydrogenase, ATP-grasp domain-containing protein, Cysteine sulfinic acid decarboxylase1-2, Taurine transporter, HSP beta 1, Sodium- and chloride-dependent glycine transporter (Meng et al., 2013; Cross et al., 2014; Eierman \& Hare, 2014).

El mix de PCR "Thermo Scientific DNA Polymerase" (Invitrogen) contenía 15 pmol por cada juego de iniciador en un volumen final de $25 \mu \mathrm{L}$ : Buffer de PCR (1X), $\mathrm{Cl}_{2} \mathrm{Mg}$ (1.5 mM), taq polimerasa $(2 \mathrm{U})$ y dNTPs $(0.2 \mathrm{mM})$. Se utilizó un termociclador (Biometra UNO thermoblock) con una programación de $95^{\circ} \mathrm{C}$ por 5 min para la desnaturalización inicial; 35 ciclos a $94{ }^{\circ} \mathrm{C}$ por $30 \mathrm{~s}$; para la desnaturalización, 50 ${ }^{\circ} \mathrm{C}$ por $45 \mathrm{~s}$ para la hibridación y $72{ }^{\circ} \mathrm{C}$ por 45 s para la polimerización. Se usó como control positivo ADN extraído a partir de branquias de Crassostrea gigas.

Extracción de proteínas a partir de tejido branquial: Fragmentos de la parte blanda de las branquias fueron extraídas a las 4, 8 y 12 horas de exposición a partir de animales expuestos y aclimatados a salinidades de 0,5 , 15 y 25 ppt, y almacenadas en PMSF $0.1 \mathrm{mM}$ (fluoruro de fenilmetilsulfonilo) a $-20{ }^{\circ} \mathrm{C}$. Para la extracción de proteínas se utilizó el método PBS 1X (buffer fosfato salino), con $1 \mathrm{mM}$ de PMSF, y se usó como agentes de precipitación TCA (ácido tricloroacetico) con acetona para luego ser resuspendido en $100 \mu \mathrm{L}$ de buffer de rehidratación (Agua grado HPLC/0.1\% TFA Ácido trifluroacetico), almacenándolo a $-20{ }^{\circ} \mathrm{C}$.

Migración en SDS page y visualización de bandas: Las proteínas purificadas fueron luego tratadas según el protocolo descrito por Shevchenko, Henrik, Jan, Jesper y Matthias (2006). Brevemente, de la muestra anterior se tomó $25 \mu \mathrm{L}$ y se mezcló con $10 \mu \mathrm{L}$ de buffer de carga (1M de Tris pH 6.8, $10 \%$ SDS, $10 \%$ glicerol, $0.02 \%$ azul de bromofenol, $0.71 \mathrm{M}$ DTT y agua bidestilada) a 2x. Se llevó a migrar en gel SDS PAGE al $12 \%$. Las bandas de interés fueron extraídas y digeridas. Las muestras fueron hidratadas con agua grado HPLC 
con TCA y se les adicionó el mismo volumen de matriz CHCA, se homogenizó cuidadosamente y fue colocada en el spot de la Opti$\mathrm{TOF}^{\circledR}$ sample plate.

\section{Análisis proteómico por espectrometría}

de masas: A través de un espectrómetro de masa MALDI TOF/TOF MS Analyzer 5800 (Applied Biosystems) en modo MS Linear High Mass Positive y MS Reflector Positive con un láser $\mathrm{Nd}$ :YAG de $\lambda$ a $335 \mathrm{~nm}$, frecuencia $200 \mathrm{~Hz}$. La placa Opti-Tof fue calibrada en un rango de $800-1800 \mathrm{~m} / \mathrm{z}$ utilizando una mezcla de calibrantes, los espectros se obtuvieron con 500 disparos con $3200 \mathrm{U}$ internas de intensidad de láser en un rango de $600-4000 \mathrm{~m} / \mathrm{z}$. En modo TOF/TOF, los iones fueron acelerados a $8 \mathrm{kV}$ y el análisis se realizó usando el programa 4000 Series Explorer versión 3.5.3 (Applied Biosystem). Se usó los iones de autólisis de la tripsina $842,510 \mathrm{~m} / \mathrm{z}$ y $2211,105 \mathrm{~m} / \mathrm{z}$ para calibración interna del equipo.

\section{RESULTADOS}

Efecto de cambios de salinidad sobre A. tuberculosa: No se reportó mortalidades en los tratamientos con salinidades de 15 a 33 ppt durante el periodo evaluado. El primer ejemplar muerto apareció al tercer día a $5 \mathrm{ppt}$; luego, la disminución de supervivencia fue progresiva, hasta que el último animal murió a los 15 días del ensayo. La mortalidad a $10 \mathrm{ppt}$ inició a los 12 días, pero fue más severa con la desaparición del $90 \%$ de los ejemplares a los cuatro días. Se observó mortalidades en los adultos mantenidos a una salinidad de $20 \mathrm{ppt}$ después de 18 días.

Desde un punto de vista de comportamiento, los animales expuestos a salinidades

CUADRO 1

Resultados de las amplificaciones de genes que intervienen en la síntesis de aminoácidos libres obtenidos por PCR en este estudio

TABLE 1

Amplification results of genes involved in the synthesis of free amino acids obtained by PCR in this study

\begin{tabular}{clcc} 
& \multicolumn{1}{c}{ Gen evaluado } & \multicolumn{2}{c}{ Especie } \\
& & A. tuberculosa & C. gigas \\
\hline 1 & Cytochrome c oxidase subunit I (COI) & Positivo & Positivo \\
2 & Aminomethyltransferase & Negativo & Positivo \\
3 & Glycine dehydrogenase & Negativo & Positivo \\
4 & Glycine hydroxymethyltransferase & Negativo & Negativo \\
5 & $\Delta-1$-pyrroline-5-carboxylate synthase & Negativo & Negativo \\
6 & $\Delta 1$-pyrroline-5-carboxylate reductase & Negativo & Positivo \\
7 & Ornithine aminotransferase & Negativo & Positivo \\
8 & Arginase & Negativo & Positivo \\
9 & Alanine transaminase & Negativo & Positivo \\
10 & Alanine-glyoxylate transaminase 2 & Negativo & Positivo \\
11 & Glutamate decarboxylase & Negativo & Positivo \\
12 & Glutamate decarboxylase & Negativo & Positivo \\
13 & Spermidine synthase & Negativo & Positivo \\
14 & Aldehyde dehydrogenase & Negativo & Positivo \\
15 & Cysteine sulfinic acid decarboxylase & Negativo & Positivo \\
16 & Cysteine sulfinic acid decarboxylase & Negativo & Positivo \\
17 & Taurine transporter & Negativo & Positivo \\
18 & Sodium- and chloride-dependent glycine transporter & Negativo & Positivo \\
19 & ATP-grasp domain-containing protein & Negativo & Positivo \\
20 & HSP beta & Negativo & Positivo \\
\hline
\end{tabular}


hipo-osmóticas extremas (5 y 10 ppt), cerraron las valvas y la filtración del alimento fue mínima. A partir del tercer día presentaron una motilidad aletargada y daño en el tejido branquial, así también, desangramiento parcial. Los animales expuestos a estrés hipo-osmótico moderado 15 ppt y 25 ppt, así como el control a 33 ppt, presentaron actividad frecuente (movilidad, alimentación), y formación de biso.

\section{Amplificación de genes osmoregulado-}

res: En este estudio se evaluó la presencia de 19 genes asociados a la osmorregulación en ostras y mejillones (Cuadro 1). De estos, se evidenció que 15 son enzimas claves del metabolismos de los FAAs (2 al 16), tres son transportadores de los principales FAAs (17 al 19), uno está relacionado al mecanismo de estrés (20), y se incluyó un control positivo que había sido utilizado en estudios anteriores sobre A. tuberculosa (1).
De todos los genes evaluados, el gen COI fue el único en ser amplificado mediante PCR en $A$. tuberculosa, lo que valida la calidad de la extracción de ADN para este molusco. Luego, 18 de los 20 genes fueron exitosamente amplificados para la muestra de $C$. gigas, incluyendo el gen COI, lo que validó también los protocolos de amplificación de la mayoría de los genes, pero $\Delta$-1-pyrroline-5-carboxylate synthase y Glycine hydroxymethyltransferase fueron los únicos que no amplificaron.

Estos resultados evidencian diferencias, al menos a nivel genómico, de los genes involucrados en la osmorregulación de $A$. tuberculosa con C. gigas.

Identificación de secuencias peptídicas: De los individuos expuestos a salinidades de 0 , 5,15 y 25 ppt durante la segunda evaluación, se extrajo proteínas a partir de las branquias. Se obtuvo un total 26 secuencias, de las cuales

CUADRO 2

Péptidos biomarcadores putativos del sistema de osmorregulación de $A$. tuberculosa sometida a diferentes condiciones de estrés identificados en la presente investigación

TABLE 2

Peptides putative biomarkers of the osmoregulation system of $A$. tuberculos $a$ under various stress conditions identified in this research

\begin{tabular}{|c|c|c|c|}
\hline Secuencia & Proteína putativa & Función & Tipo \\
\hline VSITSK & $\begin{array}{l}\text { actin-binding protein anillin- } \\
\text { like isoform X4 }\end{array}$ & $\begin{array}{l}\text { Proteínas involucradas en la localización del núcleo durante la } \\
\text { interfase y la cytokinesis. }\end{array}$ & Presencial \\
\hline DPVLFNR & $\begin{array}{l}\text { cytochrome c oxidase } \\
\text { subunit I }\end{array}$ & $\begin{array}{l}\text { Enzima clave en el metabolismo aeróbico. Involucrado en la respu- } \\
\text { esta a metales pesados en moluscos. }\end{array}$ & Presencial \\
\hline WLTSK & $\begin{array}{l}\text { sn1-specific diacylglycerol } \\
\text { lipase beta-like isoform X1 }\end{array}$ & $\begin{array}{l}\text { Involucrado en sistema nervioso. Cataliza la hidrólisis de diacilglic- } \\
\text { erol (DAG) a 2-araquidonoil-glicerol (2-AG), el endocannabinoide } \\
\text { más abundante en los tejidos. }\end{array}$ & Presencial \\
\hline$\overline{\text { GHLCR }}$ & secretin receptor-like & $\begin{array}{l}\text { La familia del receptor a secretina activa las vías de adenilato } \\
\text { ciclasa y de fosfatidil-inositol-calcio. }\end{array}$ & $\begin{array}{l}\text { Diferencial } \\
0 \text { y } 5 \mathrm{ppt}\end{array}$ \\
\hline WVDSK & $\begin{array}{l}\text { Beta-1,3-glucan-binding } \\
\text { protein }\end{array}$ & $\begin{array}{l}\text { Proteína de reconocimiento de lipopolysaccharides y } \beta \text {-1, 3-glucan, } \\
\text { que active la cascada proPO cascade en artrópodos, juega un papel } \\
\text { esencial en respuesta inmune. }\end{array}$ & $\begin{array}{l}\text { Diferencial } \\
0 \text { y } 5 \text { ppt }\end{array}$ \\
\hline TTLTSK & $\begin{array}{l}\text { integumentary mucin C.1- } \\
\text { like }\end{array}$ & Precursor de proteínas secretadas (mucus) en ranas. & $\begin{array}{l}\text { Diferencial } \\
0 \text { y } 5 \mathrm{ppt}\end{array}$ \\
\hline$\overline{\mathrm{LPPCR}}$ & $\begin{array}{l}\text { General transcription factor } \\
3 \mathrm{C} \text { polypeptide } 5\end{array}$ & $\begin{array}{l}\text { Implicado en la transcripción mediada por la ARN polimerasa } 3 \text {. } \\
\text { Componente, fuertemente asociado a la proteína TFIIIC } 2 \text { que se } \\
\text { une directamente ARNt y a promotores asociados a virus de ARN. }\end{array}$ & Presencial \\
\hline
\end{tabular}


CUADRO 2 (Continuación) / TABLE 2 (Continued)

\begin{tabular}{|c|c|c|c|}
\hline Secuencia & Proteína putativa & Función & Tipo \\
\hline NPIDFVR & $\begin{array}{l}\text { deoxynucleoside } \\
\text { triphosphate } \\
\text { triphosphohydrolase } \\
\text { SAMHD1-like }\end{array}$ & $\begin{array}{l}\text { Nucleasa de restricción que bloquea la replicación de virus en etapa } \\
\text { temprana en dendrítos y otras células mieloides. Del mismo modo, } \\
\text { suprima a retrotransposones. Podría actuar en la mediación de } \\
\text { respuestas proinflamatorias. }\end{array}$ & Presencial \\
\hline VLKKPKFDFR & $40 \mathrm{~S}$ ribosomal protein $\mathrm{S} 3 \mathrm{a}$ & Componente de la subunidad del pequeño ribosoma. & Presencial \\
\hline QTSKKMR & $\begin{array}{l}\text { tRNA (uracil-5-)- } \\
\text { methyltransferase-like } \\
\text { protein A }\end{array}$ & Metiltransferasa del ARNt. & Presencial \\
\hline CFTSK & Synaptobrevin, partial & $\begin{array}{l}\text { Pertenece a las proteínas SNARE que son componentes clave de la } \\
\text { fusión de las membranas en la exocitosis. Su función, sin embargo } \\
\text { está sujeto a un ajuste efectuado por varias proteínas reguladoras } \\
\text { que se refiere colectivamente como maestros SNARE. }\end{array}$ & $\begin{array}{l}\text { Diferencial } \\
0 \text { y } 5 \text { ppt }\end{array}$ \\
\hline VVVVSK & $\begin{array}{l}\text { cytochrome b- } 245 \text { heavy } \\
\text { chain-like, partial }\end{array}$ & $\begin{array}{l}\text { En la mitocondria de eucariotas y en procariotas aeróbicos, el cito- } \\
\text { cromo b es un componente del complejo de la cadena respiratoria II. } \\
\text { Estos complejos están involucrados en el transporte de electrones y } \\
\text { el bombeo de protones. }\end{array}$ & Presencial \\
\hline GELTSK & $\begin{array}{l}\text { chloride channel protein } \\
\text { 2-like isoform X2 }\end{array}$ & $\begin{array}{l}\text { Proteína del canal de cloruro que regulan el volumen celular; el } \\
\text { potencial de membrana, la transducción de señales y el transporte } \\
\text { trans epitelial. }\end{array}$ & Presencial \\
\hline EITSK & $\begin{array}{l}\text { adenosylmethionine synthase } \\
\text { isoform type-2-like isoform } \\
\mathrm{X} 3\end{array}$ & $\begin{array}{l}\text { Cataliza la formación de S-adenosilmetionina a partir de metionina } \\
\text { y ATP. }\end{array}$ & Presencial \\
\hline GQITSK & $\begin{array}{l}\text { glycine N-acyltransferase- } \\
\text { like [Crassostrea gigas] }\end{array}$ & $\begin{array}{l}\text { Aciltransferasa que transfiere un grupo acilo a la N-terminal de la } \\
\text { glutamina. Puede utilizar fenilacetil-CoA como donador de acilo. } \\
\text { Identificada del genoma de ostra } C \text {. gigas. }\end{array}$ & Presencial \\
\hline HGITSK & $\begin{array}{l}\text { EF-hand calcium-binding } \\
\text { domain-containing protein } \\
\text { 6-like isoform X3 } \\
\text { [Crassostrea gigas] }\end{array}$ & $\begin{array}{l}\text { El motivo de mano EF es el motivo de unión al calcio más común } \\
\text { encontrado en las proteínas, está probablemente involucrada en la } \\
\text { relajación muscular a través de su actividad de unión a calcio. }\end{array}$ & $\begin{array}{l}\text { Diferencial } \\
0 \text { y } 5 \text { ppt }\end{array}$ \\
\hline DADVSK & trichohyalin-like & $\begin{array}{l}\text { Tricohialina confiere resistencia mecánica al folículo del pelo y a } \\
\text { otros tejidos epiteliales. Está involucrado en los velos sensoriales } \\
\text { de moluscos. }\end{array}$ & $\begin{array}{l}\text { Diferencial } \\
0 \text { y } 5 \text { ppt }\end{array}$ \\
\hline ETVFDLR & $\begin{array}{l}\text { multidrug resistance- } \\
\text { associated protein 4-like }\end{array}$ & $\begin{array}{l}\text { Bomba de desintoxicación celular. Reportada para eliminación de } \\
\text { toxinas en moluscos depredadores. }\end{array}$ & $\begin{array}{l}\text { Diferencial } \\
0 \text { y } 5 \text { ppt }\end{array}$ \\
\hline ELTSK & $\begin{array}{l}\text { Tripartite motif-containing } \\
\text { protein } 3\end{array}$ & $\begin{array}{l}\text { Probablemente implicados en el tráfico vesicular a través de su } \\
\text { asociación con el complejo de CART. Identificada del genoma de } \\
\text { ostra C. gigas. }\end{array}$ & $\begin{array}{l}\text { Diferencial } \\
0 \text { y } 5 \text { ppt }\end{array}$ \\
\hline GIGCEWR & Krueppel-like factor 5 & $\begin{array}{l}\text { La familia Krüppel-como de factores de transcripción (FCE) son } \\
\text { un conjunto de proteínas de unión al ADN de dedos de zinc que } \\
\text { regulan la expresión génica. }\end{array}$ & Presencial \\
\hline LEGTNT & $\begin{array}{l}\text { heat shock } 70 \mathrm{kDa} \text { protein } \\
\text { 12A-like }\end{array}$ & $\begin{array}{l}\text { Proteínas de choque térmico (HSP) son una familia de proteínas } \\
\text { chaperonas que son producidas por las células en respuesta a la } \\
\text { exposición a condiciones de estrés y durante la curación de heridas } \\
\text { o la remodelación tisular. Varias son descriptas en bivalvos. }\end{array}$ & Presencial \\
\hline EGLTSK & $\begin{array}{l}\text { Uncharacterized protein } \\
\text { C7orf58 }\end{array}$ & Función desconocida. & Presencial \\
\hline AMVDTN & Rotatin & Grande proteína de función desconocida. & $\begin{array}{l}\text { Diferencial } \\
0 \text { y } 5 \text { ppt }\end{array}$ \\
\hline
\end{tabular}


CUADRO 2 (Continuación) / TABLE 2 (Continued)

\begin{tabular}{llll}
\multicolumn{1}{c}{ Secuencia } & \multicolumn{1}{c}{ Proteína putativa } & \multicolumn{1}{c}{ Función } & Tipo \\
LVAVRDVPRPR & gelsolin-like protein, partial & $\begin{array}{l}\text { La gelsolina es una proteína de unión a actina que es un regulador } \\
\text { clave del asambleamiento y desasambleamiento de filamentos de } \\
\text { actina. Gelsolin se encuentra intracelularmente (en citosol y mito- } \\
\text { condrias) y extracelularmente (en el plasma sanguíneo). }\end{array}$ & \\
\hline VSLTSK & pyruvate kinase & $\begin{array}{l}\text { Esta enzima está implicada en la última etapa de la glucólisis para } \\
\text { catalizar la fosforilación de una molécula de ADP en ATP. }\end{array}$ & $\begin{array}{l}\text { Diferencial } \\
0 \text { y } 5 \text { ppt }\end{array}$ \\
\hline HGLCR & $\begin{array}{l}\text { Serine/threonine-protein } \\
\text { kinase SRPK1 }\end{array}$ & $\begin{array}{l}\text { Este gen codifica una de serina arginina quinasa. La proteína se } \\
\text { localiza en el núcleo y el citoplasma. Se cree que desempeñan un } \\
\text { papel en la regulación del episaje constitutivo y alternativo. }\end{array}$ & \\
\hline
\end{tabular}

10 se expresaron únicamente en los individuos mantenidos a 0 y 5 ppt (diferencial), y 16 expresadas en todos los grupos (presencial) (Cuadro 2). De todas estas, se encontró que dos están relacionadas al mecanismo de osmorregulación en invertebrados marinos. La primera secuencia corresponde a un fragmento de una heat shock protein de $70 \mathrm{Kda}$ (HSP70), que se encontró de manera presencial en todas las muestras a diferentes salinidades, cualitativamente la HSP70 presentó una mayor expresión a 0 y 5 ppt, cuando se comparaba las intensidades de las bandas extraídas del gel de SDS Page. Por su parte, la segunda corresponde a una secuencia de seis péptidos, que pertenecen a canales de cloro (chloride channel protein), que aparece como un candidato válido del mecanismo de osmoregulación para la concha negra A. tuberculosa.

\section{DISCUSIÓN}

La comprensión de los mecanismos por los cuales los organismos pueden adaptarse a los desafíos ambientales y los factores que limitan sus capacidades de distribución, han sido durante mucho tiempo la interrogante de la biología. La supervivencia de los organismos marinos requerirá la aclimatación y adaptación a estas variantes ambientales. En el ecosistema manglar de Tumbes, donde habita el bivalvo $A$. tuberculosa existen eventos de descensos bruscos de salinidad, que suelen acontecer principalmente durante el verano con la entrada de agua dulce. En estas zonas de encuentro, la salinidad puede disminuir drásticamente en un lapso de unas horas y hasta ser completamente dulce durante las mareas bajas (Nieves et al., 2009).

El presente trabajo complementa los resultados obtenidos por Nieves et al. (2009), quienes evaluaron por 15 días al bivalvo $A$. tuberculosa, y monstraron crecimiento en adultos mantenidos entre 20 a $50 \mathrm{ppt}$ de salinidad a distintas temperaturas, concluyendo que las salinidades altas (40 a $50 \mathrm{ppt}$ ) perjudican el crecimiento, y que las condiciones óptimas de cultivo son 20 ppt y $32{ }^{\circ} \mathrm{C}$. Sin embargo, al finalizar estos experimentos, los individuos que se mantuvieron a salinidades de $20 \mathrm{ppt}$ mostraron mortalidades después de 18 días (resultados no mostrados). Por otro lado, los resultados de Diringer et al. (2012) quienes evaluaron la supervivencia de semillas de $A$. tuberculosa producidas en laboratorio y mantenidas de 45 a 0 ppt por 20 días, demostraron que la supervivencia fue severamente afectada a salinidades inferiores o igual a 20 ppt (100 $\%$ de mortalidad entre 2 a 11 días) y moderadamente afectadas a salinidad de 45 ppt $(20 \%$ de mortalidad a los 18 días). En conjunto, estos resultados demuestran que $A$. tuberculosa es una especie eurihalina. Se sugiere que la supervivencia a salinidades inferiores o iguales a 20 ppt está relacionada a la capacidad del bivalvo a reajustar su volumen celular a través de la excreción de FAAs, que y que depende de las reservas energéticas inicial de cada individuo, 
tal como lo menciona Gosling (2015). Esta hipótesis se ajusta a los resultados obtenidos con semillas de $A$. tuberculosa que poseen menos reservas o que poseen un desgaste más acelerado que un adulto.

Estudios previos han demostrado que los FAAs intracelulares son los principales contribuyentes a la regulación de la osmolaridad intracelular y del volumen celular en bivalvos (Gosling, 2015). En este trabajo se diseñó 18 marcadores moleculares asociados al sistema de regulación por FAAs, y uno a partir de la proteína de estrés (HSP70), usando las secuencias disponibles publicadas de cuatro bivalvos modelos: las ostras $C$. gigas, $C$. angulata, $C$. virginica y el mejillón $M$. galloprovincialis (Meng et al., 2013; Cross et al., 2014; Eierman \& Hare, 2014).

La utilización de dos controles positivos (COI para bivalvos y ADN de ostras) permitió confirmar que la ausencia de amplificación de los genes evaluados se debe o a diferencias genéticas en las regiones conservadas evaluadas, o a la ausencia de estos genes en $A$. tuberculosa. La primera hipótesis aparece como la más probable debido al predominio del sistema de osmorregulación mediante la utilización de FAAs en bivalvos y gasterópodos marinos; así como, porque importantes diferencias a nivel genético, en particular en los genes asociados a mecanismos de respuestas a estrés abióticos, se encontraron entre las secuencias nucleotídicas de $C$. angulata y $C$. virginica con $C$. gigas (Cross et al., 2014; Eierman \& Hare, 2014).

Existen diferentes alternativas para a futuro identificar a nivel nucleotídico los genes específicos involucrados en la osmoregulacion en $A$. tuberculosa. Los trabajos realizados a nivel genómico o transcriptómico por Cross et al. (2014), o Meng et al. (2013) y Eierman y Hare (2014) en ostras, abrieron el camino para la identificación de marcadores genéticos específicos de bivalvos, mediante la aplicación de herramientas de Next Generation Sequencing (NGS) y podrían ser aplicados a $A$. tuberculosa . Es importante resaltar un estudio realizado por Barman et al. (2012) en el camarón Macrobrachium rosenbergii, que evaluó la expresión de ARNm del crustáceo expuesto a diferentes salinidades, por transcriptómica diferencial, mediante la técnica de Hibridación Substractiva y Supresiva ( $\mathrm{SSH}$ ), que permite resaltar a los transcriptos más discretos.

Como alternativa al trabajo genético, se exploró el proteoma diferencial de A. tuberculosa sometida a diferentes salinidades, lográndose identificar a 16 proteínas "presenciales" y 10 proteínas expresadas de forma diferenciales (38.5\%) al control, mediante separación por electroforesis SDS PAGE 1D. El único trabajo similar realizado en bivalvos fue presentado por Tomanek, Zuzow, Hitt, Serafini, y Valenzuela Tomanek, (2012) que compararon la respuesta al estrés hipo-osmótico en dos especies de mejillón. A pesar de que este equipo obtuvo más proteínas aisladas gracias a un sistema de separación SDS PAGE 2D, la proporción de proteínas diferenciales fue de $29 \%$ para $M$. galloprovincialis y $39 \%$ para M. trossulus, lo que está dentro del rango del presente estudio.

La familia de HSP es comúnmente encontrada en moluscos sometidos a estrés abióticos y ha sido reiteradamente propuesta como biomarcador (Gracey et al., 2008; Yang et al., 2014; Patterson, Boettcher, \& Carmichael, 2014; Liu, He, Chi, \& Lv, 2015; Shiel, Hall, Cooke, Robinson, \& Strugnell, 2015). En este trabajo se intentó sin éxito amplificar por PCR una región conservada de esta chaperona, mientras que esta proteína pudo ser detectada mediante proteómica, esto se debe al carácter muy variable de HSP70 que presenta mucha variabilidad intra e inter específica, tal como lo menciona Yang et al. (2014).

El canal a cloro encontrado en $A$. tuberculosa, presentó grandes similitudes con una proteína de $C$. gigas (chloride channel protein 2-like isoform X2) sometida a condiciones de estrés de salinidad (Damiens et al., 2006). Estos canales cumplen una variedad de importantes roles celulares y fisiológicos entre los que se encuentran la regulación del $\mathrm{pH}$, regulación del volumen en la homeostasis, transporte de solutos orgánicos, migración celular y diferenciación por lo que parece como un candidato 
válido del mecanismo de osmoregulación para A. tuberculosa.

La no amplificación de los genes evaluados por PCR revela diferencias genéticas entre A. tuberculosa y los bivalvos previamente estudiados (C. gigas, C. angulata, O. edulis y M. galloprovincialis), sin poder determinar si estas corresponden a diferencias nucleotídicas de las regiones conservadas, o por la ausencia de estos genes en $A$. tuberculosa.

Las secuencias aminoacídicas obtenidas por espectrometría de masas identificaron HSP 70, canal de cloro, y al piruvato kinasa como posibles biomarcadores del sistema de osmorregulación en $A$. tuberculosa, y permitirán diseñar iniciadores específicos para la puesta en marcha de futuros programas de mejoramiento genético de este importante recurso.

\section{AGRADECIMIENTOS}

El presente estudio fue posible gracias al aporte de financiamientos por parte de la Universidad Nacional de Tumbes a través del proyecto CANON Resolución $\mathrm{N}^{\circ}$ 00723-2014/UNT-R., K.P y V.C. recibieron una beca de CONCYTEC (CONVENIO DE GESTIÓN $\mathrm{N}^{\circ}$ 015-2013- FONDECYT, y R.P.N ${ }^{\circ}$ 206-2013-CONCYTEC-P.), y B.D de la Escuela Doctoral Franco-Peruana en Ciencias de la Vida.

\section{RESUMEN}

La concha negra Anadara tuberculosa es una especie emblemática del ecosistema manglar que está actualmente en condición vulnerable. El desarrollo de su acuicultura requiere identificar biomarcadores moleculares, en particular asociados al estrés por salinidad en mira al inicio de programas de mejoramiento genético. Se recolectaron ejemplares de Anadara tuberculosa del manglar colindante a la Bahía de Puerto Pizarro (Tumbes, Perú) entre enero 2015 y febrero 2016. Estos individuos fueron sometidos a condiciones de estrés hipo-osmótico (extremo: 5 y 10 ppt); (moderado: 15 y 25 ppt) y sin estrés (grupo control: $33 \mathrm{ppt}$ ) por 16 días después de haber sido separados en grupos de diez animales y por triplicado. La presencia de biomarcadores del estrés por salinidad fue evaluada a nivel genético con la detección por PCR de 19 genes reportados como actores claves de la osmorregulación en bivalvos como ostras y mejillones y a nivel proteomico con la secuenciación de péptidos expresados en tejidos de animales expuestos a diferentes salinidades por espectrometría de doble masa. Ninguno de los marcadores genéticos probados pudo ser amplificado por PCR lo que sugiere que A. tuberculosa presente diferencias genéticas significativas en comparación con otros moluscos. El análisis proteómico realizado por MALDI TOF/TOF a nivel de tejido branquial de $A$. tuberculosa permitió identificar 26 péptidos expresados de formas presenciales y diferenciales a las diferentes salinidades evaluadas, resaltando posibles marcadores como la HSP70 y una proteína transmembrana de transporte de cloruro que están relacionadas con la adaptación a la salinidad. Estas secuencias aminoacídicas permitirán diseñar iniciadores nucleotidicos específicos a $A$. tuberculosa para la puesta en marcha de futuras investigaciones en ecofisiología de este importante recurso.

Palabras clave: Anadara tuberculosa, osmorregulación, salinidad, adaptación, manglar.

\section{REFERENCIAS}

Artigaud, S., Lacroix, C., Richard, J., Flye-Sainte-Marie, J., Bargelloni, L., \& Pichereau, V. (2015). Proteomic responses to hypoxia at different temperatures in the great scallop (Pecten maximus). PeerJ, 3, e871. doi: $10.7717 /$ peerj. 871

Barman, H. K., Patra, S. K., Das, V., Mohapatra, S. D., Jayasankar, P., Mohapatra, Ch., Mohanta, R., Panda, R. P., \& Rath. S. N. (2012). Identification and Characterization of Differentially Expressed Transcripts in the Gills of Freshwater Prawn (Macrobrachium rosenbergii) under Salt Stress. The Scientific World Journal, doi:10.1100/2012/ 149361

Cruz, R., Fonseca, R. \& Chavarría-Solera, F. (2012). Comparación de la composición química proximal de la carne de A. tuberculosa y A. similis (Bivalvia: Arcidae) de Chomes, Puntarenas, Costa Rica. Revista de Ciencias Marinas y Costeras, 4, 95-103. Recuperado de http://www.revistas.una.ac.cr/index.php/revmar/ article/view/4801

Cross, I., Merlo, M., Rodríguez, M. E., Portela-Bens, S., \& Rebordinos, L. (2014). Adaptation to abiotic stress in the oyster Crassostrea angulata relays on genetic polymorphisms. Fish Shellfish Immunology, 41(2), 618-24. doi: 10.1016/j.fsi.2014.10.011

Damiens, G., Mouneyrac, C., Quiniou, F., His, E., GnassiaBarelli, M., \& Roméo, M. (2006). Metal bioaccumulation and metallothionein concentrations in larvae of Crassostrea gigas. Environmental Pollution, 140(3), 492-9, doi: 10.1016/j.envpol.2005.08.006

Diringer, B., Vasquez, R., Moreno, V., Pretell, K., \& Sahuquet, M. (2012). Peru Project Studies Blood cockles for stock enhancement, Aquaculture. Global 
Aquaculture Advocate, Ju-Agt, 48-50. Recuperado de http://pdf.gaalliance.org/pdf/GAA-DiringerJuly12.pdf.

Eierman, L. E., \& Hare, P. M. (2014). Transcriptomic analysis of candidate osmoregulatory genes in the eastern oyster Crassostrea virginica. Bio Med Central Genomics, 15, 503, doi: 10.1186/1471-2164-15-503

Espinosa, S. G., Delgado, M. H., Orobio, B. R., MejíaLadino, L. \& Gil-Agudelo, D. (2010). Estado de la población y valoración de algunas estrategias de conservación del recurso piangua Anadara tuberculosa (Sowerby) en sectores de Bazán y Nerete, costa pacífica nariñense de Colombia. Boletín Investigación Marina Costera, 39(1), 161-176. Recuperado de http://www.scielo.org.co/pdf/mar/v39n1/ v39n1a09.pdf

Gardes, M., \& Bruns, T. D. (1993). ITS primers with enhanced specificity for basidiomycetes - applications to the identification of mycorrhizae and rusts. Molecular Ecology, 2, 113-118. Recuperado de https://nature.berkeley.edu/brunslab/papers/ gardes1993.pdf

Gosling, E. (2015). Marine Bivalve Molluscs (2nd ed). Oxford, U.K.: Editorial offices.

Gracey, Y., Chaney, M. L., Boomhower, J. P., Tyburczy, W. R., Connor, K., \& Somero, G. N. (2008). Rhythms of Gene Expression in a Fluctuating Intertidal Environment. Current Biology, 18, 1501-1507, doi:10.1016/j. cub.2008.08.049

Liu, H., He, J., Chi, C., \& Lv, Z. (2015). Identification and analysis of HSP70 from Sepiella maindroni under stress of Vibrio harveyi and $\mathrm{Cd}^{2+}$. Epub 1, 572(1), 146-52. Recuperado de http://dx.doi.org.sci-hub. bz/10.1016/j.gene.2015.07.056

Lucero, C., Cantera, J., \& Neira, R. (2012). Pesquería y crecimiento de la piangua (Arcoida: Arcidae) Anadara tuberculosa en la Bahía de Málaga del Pacífico colombiano, 2005-2007. Revista Biología Tropical, 60(1), 203-217.

Mendoza, O. \& Peralta, T. (2004). Primeros ensayos sobre reproducción inducida y obtención de semillas de Anadara tuberculosa (Sowerby, 1833) en laboratorio. Revista Manglar, 2(1), 87-94.

Meng, J., Zhu, Q., Zhang, L., Li, Ch., Li, L., She, Z., Huang, B., \& Zhang, G. (2013). Genome and Transcriptome Analyses Provide Insight into the Euryhaline Adaptation Mechanism of Crassostrea gigas. PLoS One, 8(3), e58563, doi: 10.1371/journal. pone. 0058563
Nieves, M., Román, J. C., Piña, P., Medina, A., Leal, S., Miranda, A., \& Muñoz, G. (2009). Balance energético de Anadara tuberculosa (SOWERBY, 1833) a diferentes temperaturas. Revista de Investigaciones Marinas, 30, 135-144. Recuperado de http://www. rim.uh.cu/index.php/IM/article/viewFile/131/130

Ordinola, E., Montero, P., Alemán, S., \& Llanos, J. (2007). Prospección del recurso concha negra (Anadara tuberculosa) en los manglares de Tumbes. Tumbes: IMARPE.

Patterson, K., Boettcher, A., \& Carmichael, R. (2014). Biomarkers of dissolved oxygen stress in oysters: a tool for restoration and management efforts. PLoS One, 12, 9, e104440. doi: 10.1371/journal.pone.0104440

Rocher, B., Bultelle, F., Chan, Ph., Le Foll, F., Letendre, J., Monsinjon, T., Olivier, S., ..., \& Knigge, T. (2015). 2-DE Mapping of the Blue Mussel Gill Proteome: The Usual Suspects Revisited. Proteomes, 3(1), 3-41, doi: $10.3390 /$ proteomes 3010003

Shevchenko, A., Henrik, T., Jan, H., Jesper, V., \& Matthias, M. (2006). In-gel digestion for mass spectrometric characterization of proteins and proteomes. Nature Protocols, 6(1), 2856-2860. doi: 10.1038/ nprot. 2006.468

Shiel, B., Hall, N., Cooke, I., Robinson, N., \& Strugnell, J. (2015). De novo characterisation of the greenlip abalone transcriptome (Haliotis laevigata) with a focus on the heat shock protein 70 (HSP70) family. Marine Biotecnology (NY), 17(1), 23-32. doi: 10.1007/ s10126-014-9591-y

Tomanek, L., Zuzow, M. J., Hitt, L., Serafini, L., \& Valenzuela, J. J. (2012). Proteomics of hyposaline stress in blue mussel congeners (genus Mytilus): implications for biogeographic range limits in response to climate change. The Journal of Experimental Biology, 215, 3905-3916. doi:10.1242/jeb.076448

Yang, C., Wang, L., Wang, J., Jiang, Q., Qiu, L., Zhang, H., \& Song, L. (2014). The polymorphism in the promoter of HSP70 gene is associated with heat tolerance of two congener endemic bay scallops (Argopecten irradians irradians and A. i. concentricus). PLoS One, 9(7), e102332. Recuperado de https://www. ncbi.nlm.nih.gov/pmc/articles/PMC4100766/pdf/ pone.0102332.pdf

Zhao, X., Yu, H., Kong, L., \& Li, Q. (2012). Transcriptomic responses to salinity stress in the pacific oyster Crassostrea gigas. PLoS One, 7(9), e46244, doi: 10.1371/journal.pone.0046244 